\title{
AN ADDITION THEOREM FOR LEBESGUE AREA ${ }^{1}$
}

HERBERT FEDERER

Let $X$ be the space of a finite 2-dimensional cell complex $K$, in the sense that $K$ is a finite set of compact topological cells of dimensions $\leqq 2$ with disjoint interiors, with union $X$, and such that the boundary of each element of $K$ is the union of certain lower dimensional elements of $K$. Also let $f$ be a continuous map of $X$ into Euclidean $n$-space, with $n \geqq 2$. Observing that $X$ is finitely triangulable and designating $k$-dimensional Lebesgue area by $L_{k}$, we may consider the area $L_{2}(f)$, the area $L_{2}(f \mid A)$ of the restriction of $f$ to each 2-cell $A$ in $K$, and the length $L_{1}(f \mid B)$ of the restriction of $f$ to each 1-cell $B$ in $K$. We shall prove the following additivity theorem:

If $L_{1}(f \mid B)<\infty$ for each 1 -cell $B$ in $K$, then

$$
L_{2}(f)=\sum_{A} L_{2}(f \mid A) \text {, }
$$

where the summation extends over all 2-cells $A$ in $K$.

This result is the solution of a classical problem raised by the original work of Lebesgue [1]. It provides one of the basic tools for the extension of the whole theory of Lebesgue area to continuous maps of a 2-manifold into $n$-space, which is presently being carried out by Paul Slepian.

For the special case in which $X$ is a 2-cell the additivity theorem follows immediately (see [2, page 506] for $n \leqq 3$ ) from the known equality between the Lebesgue area of $f$ and any one of several areas which may be defined in terms of the Lebesgue areas of the 2-dimensional projections of $f$. In fact it is obvious that the addition formula holds with $L_{2}$ replaced by the 2-dimensional integralgeometric stable area (see [3, page 319]), which agrees (according to $[4,7.16]$ ), with $L_{2}$ for maps of a 2-cell into $E_{n}$.

Next consider the special case in which $f$ maps each 1 -cell in $K$ onto the union of finitely many straight line segments in $n$-space. Then the $f$ image of the 1-skeleton of $K$ is 2-removable (by [4, 6.26, $6.27,6.30]$ ), hence $L_{2}(f)$ is the supremum of $L_{2}(f \mid Y)$ over all those finitely triangulable subsets $Y$ of $X$ which do not meet the 1-skeleton of $K$. Now 1955.

Presented to the Society, February 27, 1954; received by the editors January 26,

1 This work was supported in part by a research grant from the National Science Foundation. 


$$
L_{2}(f \mid Y)=\sum_{A} L_{2}(f \mid Y \cap A)
$$

for any such set $Y$, because the intersections of $Y$ with the 1-cells in $K$ are disjoint. Hence passage to the supremum yields the addition formula for the present special case.

The general additivity theorem may be derived from the preceding two special cases as follows:

Suppose $\epsilon>0$.

For each 1-cell $B$ in $K$ the curve $f \mid B$ is rectifiable, by hypothesis, and we can therefore select ${ }^{2}$ a homeomorphism $h_{B}$ which maps the interval $\{t \mid 0 \leqq t \leqq 1\}$ onto $B$ in such a way that

$$
\left|\left(f \circ h_{B}\right)\left(t_{2}\right)-\left(f \circ h_{B}\right)\left(t_{1}\right)\right| \leqq\left[L_{1}(f \mid B)+\epsilon\right] \cdot\left(t_{2}-t_{1}\right)
$$

whenever $0 \leqq t_{1} \leqq t_{2} \leqq 1$.

For each 2-cell $A$ in $K$ we let $B_{1}(A), B_{2}(A), \cdots, B_{\mu(A)}(A)$ be those 1-cells in $K$ which are contained in the boundary of $A$, ordered in such a way that we can select a homeomorphism $h_{A}$ of the circular $\operatorname{disc}\{z|| z \mid \leqq 1\}$ onto $A$ for which $h_{A}\left(e^{i \theta}\right)=h_{B_{j}(A)}[\mu(A) \theta / 2 \pi-j+1]$ whenever $(j-1) / \mu(A) \leqq \theta / 2 \pi \leqq j / \mu(A)$ and $j=1,2, \cdots, \mu(A)$.

Associating with each 1-cell $B$ in $K$ the number $\nu(B)$ of 2-cells $A$ in $K$ such that $B$ is contained in the boundary of $A$, we choose an integer

$$
q>\epsilon^{-1} \sum_{B} \nu(B)\left[L_{1}(f \mid B)+\epsilon\right]^{2},
$$

where the summation extends over all 1-cells $B$ in $K$, and construct a continuous map $g$ of $X$ into $n$-space which agrees with $f$ at the vertices of $K$ and which is characterized by the following two properties:

If $B$ is a 1 -cell in $K$, then $g \circ h_{B}$ is the polygonal function with $q+1$ vertices which agrees with $f \circ h_{B}$ at $0,1 / q, 2 / q, \cdots, 1$.

If $A$ is a 2 -cell in $K$, then

$$
\left(g \circ h_{A}\right)\left(r e^{i \theta}\right)=(1+q)(1-r)\left(f \circ h_{A}\right)\left(e^{i \theta}\right)+[r(q+1)-q]\left(g \circ h_{A}\right)\left(e^{i \theta}\right)
$$

in case $q /(q+1) \leqq r \leqq 1$, and

$$
\left(g \circ h_{A}\right)\left(r e^{i \theta}\right)=\left(f \circ h_{A}\right)\left(\frac{q+1}{q} r e^{i \theta}\right)
$$

in case $0 \leqq r \leqq q /(q+1)$.

Since $g$ maps each 1 -cell in $K$ onto the union of finitely many

2 In case $B=\{t \mid 0 \leqq t \leqq 1\}$, let $h_{B}$ be the inverse of the function which maps $t$ onto $\left(L_{1}(f \mid\{u \mid 0 \leqq u \leqq t\})+\epsilon t\right) /\left(L_{1}(f \mid B)+\epsilon\right)$. 
straight line segments in $n$-space, the addition formula holds with $f$ replaced by $g$, so that

$$
L_{2}(g)=\sum_{A} L_{2}(g \mid A) .
$$

Moreover for each 2-cell $A$ in $K$ we may apply the addition formula with $f, X$, and $K$ replaced by $g \circ h_{A},\{z|| z \mid \leqq 1\}$, and a complex whose 2-cells are the circular disc

$$
D=\left\{z|| z \mid \leqq \frac{q}{q+1}\right\}
$$

and the annular sectors

$$
S_{j}=\left\{r e^{i \theta} \mid \frac{q}{q+1} \leqq r \leqq 1 \text { and } \frac{j-1}{\mu(A)} \leqq \frac{\theta}{2 \pi} \leqq \frac{j}{\mu(A)}\right\}
$$

corresponding to $j=1,2, \cdots, \mu(A)$; we find that

$$
L_{2}(g \mid A)=L_{2}\left(g \circ h_{A}\right)=L_{2}\left(g \circ h_{A} \mid D\right)+\sum_{j=1}^{\mu(A)} L_{2}\left(g \circ h_{A} \mid S_{j}\right) .
$$

Evidently

$$
L_{2}\left(g \circ h_{A} \mid D\right)=L_{2}\left(f \circ h_{A}\right)=L_{2}(f \mid A) .
$$

For each $j$ we map the square

$$
Q=\{(s, t) \mid 0 \leqq s \leqq 1 \text { and } 0 \leqq t \leqq 1\}
$$

homeomorphically onto $S_{j}$ by mapping $(s, t)$ onto

$$
\frac{q+s}{q+1} \exp \left[\frac{i 2 \pi}{\mu(A)}(t+j-1)\right]
$$

thus transforming $g \circ h_{A} \mid S_{j}$ into a function on $Q$ which maps $(s, t)$ onto

$$
(1-s)\left[f \circ h_{B_{j}(A)}\right](t)+s\left[g \circ h_{B_{j}(A)}\right](t) .
$$

At almost all points $(s, t)$ in $Q$ the two partial derivatives of this Lipschitzian function are the vectors

$$
\left[g \circ h_{B_{j}(A)}\right](t)-\left[f \circ h_{B_{j}(A)}\right](t)
$$

and

$$
(1-s)\left[f \circ h_{B_{j}(\boldsymbol{A})}\right]^{\prime}(t)+s\left[g \circ h_{B_{j}(A)}\right]^{\prime}(t),
$$

whose norms are less than or equal to

$$
q^{-1}\left(L_{1}\left[f \mid B_{j}(A)\right]+\epsilon\right),
$$




$$
L_{1}\left[f \mid B_{j}(A)\right]+\epsilon
$$

respectively. Therefore the Jacobian integral over $Q$, which equals $L_{2}\left(g \circ h_{A} \mid S_{j}\right)$, does not exceed

$$
q^{-1}\left(L_{1}\left[f \mid B_{j}(A)\right]+\epsilon\right)^{2}
$$

It follows that

$$
L_{2}(g \mid A) \leqq L_{2}(f \mid A)+q^{-1} \sum_{j=1}^{\mu(A)}\left(L_{1}\left[f \mid B_{j}(A)\right]+\epsilon\right)^{2} .
$$

Summing over all 2-cells $A$ in $K$ we obtain the inequality

$$
L_{2}(g) \leqq \sum_{A} L_{2}(f \mid A)+\epsilon .
$$

Inasmuch as $g$ is (uniformly) close to $f$ for large $q$, and in view of the arbitrary nature of $\epsilon$, we infer that

$$
L_{2}(f) \leqq \sum_{A} L_{2}(f \mid A)
$$

We complete the proof by observing that the opposite inequality is an obvious consequence of $[4,6.25]$ and of the fact that, for each 2-cell $A$ in $K, L_{2}(f \mid A)$ is the supremum of $L_{2}(f \mid C)$ over all 2-cells $C$ contained in the interior of $A$.

\section{BIBLIOGRAPHY}

1. H. Lebesgue, Intégrale, longueur, aire, Annali di Matematica Pura ed Applicata vol. 7 (1902) pp. 231-359.

2. T. Rad6, Length and area, Amer. Math. Soc. Colloquium Publications, vol. 30, 1948.

3. H. Federer, Measure and area, Bull. Amer. Math. Soc. vol. 58 (1952) pp. 306378.

4. - On Lebesgue area, Ann. of Math. vol. 61 (1955) pp. 289-353.

BRown UNIVERSITY 